\title{
El Aumento de la Masa Inercial; Einstein y las Coordenadas
}

Inertial Mass Increase: Einstein and the coordinates system

\author{
Favio Ernesto Cala Vitery ${ }^{1}$ \\ Departamento de Filosofía, \\ Universidad Autónoma de Barcelona
}

\section{Resumen}

Durante los años de gestación de la Teoría General de la Relatividad (TGR) y de la subsiguiente cosmología relativista, Albert Einstein esperaba que su teoría de gravitación satisficiera las ideas de Mach sobre la inercia. Para esto calculó un par de efectos que debían garantizar la consonancia de TGR con el origen material de la inercia à la Mach. Estos efectos fueron: (A) El aumento de la masa inercial cuando se aglomera materia en su vecindad y (B) el arrastre de los marcos inerciales. En este articulo se hace un estudio histórico-conceptual del primero(A). Mientras que el arrastre es considerado como una predicción real de TGR, el aumento de la masa inercial ha llegado a desecharse como un artificio de coordenadas no impugnable a la variedad espacio-temporal estudiada. Una revisión técnica con clarificaciones conceptuales es presentada.

Palabras claves: masa, inercia, relatividad

\begin{abstract}
While creating his General Theory of Relativity (GR) an the subsequent Relativistic Cosmology, Einstein hoped that his new theory of gravitation would conform to Mach's ideas on inertia. In doing so, he calculated two important effects that should ensure GR's accordance with the material origin of inertia. These effects were: (A) the increase of the inertial mass of a body when matter is piled up in its neighbourhood and $(B)$ the dragging of inertial frames. A historical and conceptual survey of $(A)$ is presented. While frame dragging is considered a real prediction of $\mathrm{GR}$, the increase if inertial mass has been rejected as a coordinate effect. A technical survey with conceptual insights is presented.
\end{abstract}

Key words: relativity, inertia, mass.

\footnotetext{
1 Msc. Física Teórica (UIS).

Msc. Historia de la Ciencia (UAB).

Candidato a Doctor en Filosofía (UAB).

Coinvestigador Grupo Matrix (UMNG).

e-mail: calavitery@yahoo.com.mx
} 


\section{INTRODUCCIÓN}

En 1883 Ernst Mach, propuso que la inercia de un cuerpo debía tener fuentes materiales. La materia estelar, en lugar del invisible y epistemológicamente indeseable espacio absoluto de Newton, debería originar las fuerzas inerciales de la mecánica. La idea, durante largo tiempo, se convirtió en un fuerte estímulo para Einstein. Desde 1907, cuando empezó a trabajar en su teoría de gravitación (e inercia), Einstein ya esperaba que sus concepciones sobre la relatividad de la inercia se resolvieran en concordancia con las ideas de Mach sobre la misma. Por esto desde la relatividad de la inercia pudo pronosticar algunos efectos que, a su entender, cabria esperar en el tejido de una teoría ajustada a los requerimientos de Mach sobre la misma ${ }^{2}$. Estos efectos fueron: el aumento de la masa inercial cuando se acumula materia en la vecindad de un cuerpo y lo que hoy conocemos como el arrastre de los marcos inerciales debido a la aceleración de la materia de su entorno.

Estos efectos Machianos nacieron de la fuerte convicción de Einstein en la relatividad de la inercia y su supuesta sintonía con las ideas de Mach sobre la misma. Por esto, éstos fueron concebidos durante el período de gestación de TGR como pruebas de su éxito en relación con las ideas de Mach. Pero ya en el contexto de TGR, Einstein presentó los cálculos de los mismos en 1922, en una época en que empezaba a declinar del principio de Mach y el difícil tinglado de la relatividad de la inercia desplazaba su protagonismo como principal fuerza motriz de sus investigaciones en favor del frustrado proyecto de una teoría de campo unificado. Con todo, los efectos Mach-Einstein ocupan un lugar importante en la historia y el presente de la relatividad general y de la cosmología relativista. El arrastre de marcos inerciales es considerado como una predicción física real que sigue despertando interés ya sea como posible prueba experimental de la precisión predictiva de TGR o como la mas efectiva realización de las ideas de Mach en la teoría de Einstein. Empero el aumento de la masa inercial de un cuerpo ante la presencia de materia en su vecindad es considerado como un engañoso efecto de coordenadas y su relación con las ideas de Mach es no menos que controversial, como controversial sigue siendo el

Principio de $\mathrm{Mach}^{3}$. A continuación se presentaran las primeras consideraciones que, ya en el contexto de TGR, se hicieron sobre los efectos Machianos.

\footnotetext{
${ }^{2}$ Einstein entendía, siguiendo a Mach, la relatividad de la inercia como el postulado de que la inercia debería entenderse no como la resistencia de un cuerpo a la aceleración con respecto al espacio (absoluto vacío) sino como la resistencia al movimiento relativo de un cuerpo con respecto a la totalidad de la materia circundante.

${ }^{3}$ El principio de Mach ha recibido diversas formulaciones, pero en manos de Einstein se trataba del requerimiento de que la métrica $\left(g_{\mu v}\right)$ estuviera completamente determinada por el tensor de energía-momento $\left(\mathrm{T}_{\mu \mathrm{v}}\right)$.
} 


\section{SOBRE LOS CÁLCULOS DE EINSTEIN}

En Mayo de 1921 dentro de un conjunto de conferencias ofrecidas en la Universidad de Princeton, Einstein mostraba los cálculos correspondientes a los esperados efectos machianos en TGR. Einstein, al intentar estudiar la influencia relativa del movimiento de materia y de la presencia de materia misma, en la inercia de los cuerpos realizó sus cálculos dentro de su propio esquema de las ecuaciones perturbativas de campo débil. Estas suponen que la geometría del espacio-tiempo estudiado, exhibe aproximadamente la geometría de Minkowski(o la Galilena-Euclideana, como él hubiera preferido llamarla) y que la presencia de materia perturba débilmente esta geometría. Al final se tiene una geometría cuasieuclideana que depende fuertemente de las condiciones de frontera. Que Einstein se permitiera utilizar este esquema para estudiar las ideas de Mach sobre el origen de la inercia hubiera resultado insólito pocos años atrás cuando el principio de Mach se erigía como emblema de su pensamiento. Pero ahora, desde un enfoque local, y sin olvidar que el esquema perturbativo simplifica considerablemente las ecuaciones de campo, el escenario es diferente ${ }^{4}$.

Para investigar la relatividad de la inercia Einstein procedió a calcular la métrica del espacio-tiempo debida una distribución de partículas materiales (correspondientes a una densidad de masa $\sigma$ ) que tienen pequeñas velocidades dxi/ds. El movimiento (aceleración) de la materia debería inducir el arrastre sobre una partícula de prueba y la distribución material debería producir el aumento de la inercia(masa inercial)de la misma. Estos efectos serían visibles al calcular las ecuaciones de movimiento, las ecuaciones geodésicas para una partícula de prueba en un espacio-tiempo que representa la acción del campo gravitatorio generado por la mencionada distribuci'on de materia en movimiento. Para esto la métrica que Einstein encontró -dentro de la aproximación de campo débil- fue la siguiente ${ }^{5}$ :

$$
\begin{gathered}
g_{00}=1-\frac{k}{4 \pi} \int \frac{\sigma d V}{r}, \\
\mathrm{~g}_{0 \mathrm{i}}=\frac{\mathrm{k}}{2 \pi} \int \frac{\sigma \mathrm{dx} / \mathrm{ds}}{\mathrm{r}} \mathrm{dV},
\end{gathered}
$$

\footnotetext{
${ }^{4}$ Aquí, por enfoque local me refiero al estudio de la influencia de la materia cercana en la dinámica de partículas. Esto en contraste con el enfoque global o cosmológico que Einstein pretendió años antes (1912-1918) cuando el principio de Mach le obligaba a considerar la totalidad de la materia cósmica en la dinámica.

${ }^{5}$ Aquí se ha reemplazando el tiempo imaginario $x_{4}$ que Einstein utilizó, por el tiempo real $x_{0}=i x_{4}$, pero esto sólo es un ajuste a una notación mas convencional.
} 


$$
\mathrm{g}_{\mathrm{ij}}=-\partial_{\mathrm{ij}}\left(1+\frac{k}{4 \pi} \int \frac{\sigma d V}{r}\right)
$$

Donde estas componentes métricas son válidas a primer orden en $k \int \sigma d V / r$ y $d x i / d s$, dentro de la aproximación perturbativa empleada por Einstein. En este campo (espacio-tiempo) la ecuación de las geodésicas,

se escribe así:

$$
\frac{d^{2} x^{\mu}}{d s^{2}}+\Gamma_{\alpha \beta}^{\mu} \frac{d x_{\alpha}}{d s} \frac{d x_{\beta}}{d s}=0,
$$

$$
\frac{d}{d x^{0}}[(1+\bar{\sigma}) \vec{v}]=\nabla \bar{\sigma}+(\nabla \times \overrightarrow{\mathrm{N}}) \times \vec{v},
$$

donde

$$
\begin{gathered}
\vec{v} \equiv d \vec{x} / d s, \\
\vec{\sigma} \equiv \frac{k}{8 \pi} \int \frac{\sigma d V}{r}, \\
\overrightarrow{\mathrm{N}} \equiv \frac{k}{2 \pi} \int \frac{\sigma \vec{v}}{r} d V,
\end{gathered}
$$

La ecuación geodésica (1) que, insisto, describe el movimiento de una partícula de prueba ante la acción de gravedad e inercia (solamente) fue interpretada por Einstein de la siguiente manera (cito)(Einstein 1922, p 102):

1. The inert mass is proportional to $1+\vec{\sigma}$, and therefore increases when ponder able masses approach the test body.

2. There is an inductive action of accelerated masses, of the same sign, upon the test body. This is the term $\frac{\partial \overrightarrow{\mathrm{N}}}{\partial x^{0}}$

3. A material particle, moving perpendicularly to the axis inside a rotating hollow body, is deflected in the sense of the rotation (Coriolis field). The centrifugal action, mentioned above, inside a rotating hollow body, also follows from the theory, as has been shown by Thirring.

Como se ve, los esperados efectos machianos encontraban (o al menos así lo creyó Einstein) forma predictiva explícita en el contexto de TGR. Con esto Einstein cerraba el largo camino emprendido, al amparo de las ideas de Mach sobre la inercia, desde que gravedad envolvieran la ilusión de concebir una auténtica 
Teoría General de la Relatividad. En todo caso tras mencionar sus conclusiones se lee a un Einstein que aún parece esperanzado (aunque sin poner las manos en el fuego) en que lo local (representado por dichos efectos) pueda traducirse a nivel cosmológico en la restauración del principio de Mach. Leo (Einstein 1922, p103):

Although all of this effects are inaccessible to experiment, because $\mathrm{K}$ is so small, nevertheless they certainly exist according to the general theory of relativity. We must see in them a strong support for Mach's ideas as to the relativity of all inertial actions. If we think this ideas consistently through

the end we must expect the whole inertia, that is the whole guv-field, to be determined by the matter of the universe, and not mainly by the boundary conditions at infinity.

De cualquier forma, esta incierta esperanza en la probable restauración del principio de Mach fue diluyéndose en el pensamiento de Einstein. Principalmente porque desde principios de los años 20 Einstein se había visto fuertemente atraído por la idea original de Hermann Weyl de construir una teoría de campo unificado que, mediante una apropiada generalización del tensor métrico, permitiera fundir el campo electromagnético y la gravitación en un solo campo. Un único campo al que resultaría (de resultar) difícil sustraer su condición ontológica como entidad física real - y fundamental- y que al ser visto así (como llegó a verlo Einstein) podía resultar conflictivo con un principio de Mach al que ulteriormente llegaría a juzgar como una especie de atavismo propio de los años en que las masas ponderables y las fuerzas a distancia dominaban la forma de entender la realidad física ${ }^{6}$. Con todo, estas palabras optimistas de la conferencia de Princeton, que habían sido publicadas por primera vez en 1922 bajo el título de The Meaning of Relativity, fueron conservadas hasta la quinta edición del libro (la última revisada por el propio Einstein que curiosamente ya incluía un apéndice sobre la teoría de campo relativista). Y menciono esto porque esta edición, de 1955, caería en manos de Carl Brans y el interés de este último por el tema del principio de Mach en el contexto de la teoría general de la relatividad es fundamental para redondear la historia que nos ocupa y también para alimentar el renovado interés que, pese a las dubitativas renuencias finales de Einstein, ha suscitado el tema principalmente a partir de los años 60. A continuación se comentarán sucintamente las observaciones de Brans sobre los cálculos de Einstein.

\section{SOBRE LOS CÁLCULOS DE BRANS}

Para abrir estas líneas reparemos en que el arrastre de los marcos inerciales parece una consecuencia directa de las ideas de Mach sobre la inercia y no sería atrevido suponer que, en buena medida, por esto algunos, como Friedlander (1896) y Föppl (1904), se anticiparon a Einstein al entretener algún tipo de consideración interesante al respecto. Pero al lado de este efecto machiano, el aumento de la masa inercial por aglomeración de materia en la vecindad de un cuerpo parece una preedición exclusiva, que yo sepa, de Einstein.

\footnotetext{
${ }^{6}$ Véase por ejemplo, Einstein 1949, p. 29.
} 
Sin ser el primero, Brans dedicó parte de su trabajo de tesis doctoral a poner algunas objeciones que resultarían perentorias para el ya discutible aumento de la masa inercial en TGR. El resultante artículo, que resumía buena parte de su trabajo de tesis doctoral, fue publicado en 1962 en Physical Review llevando por título el de Mach's Principle and the Locally Measured Gravitational Constant in General Relativity. Allí se anticipaban algunas de las ideas germinales que, en compañía de R.H. Dicke, llevaron a Brans a postular modificaciones importantes a las ecuaciones de campo de Einstein para ajustarlas al principio de Mach. Pero ahora nos ocupamos brevemente de las implicaciones de sus correcciones a los cálculos que habían llevado a Einstein a predecir felizmente el aumento de la masa inercial en TGR. Recordemos que Einstein había asociado el término $1+\vec{\sigma}$ de su ecuación de las geodésicas (1) con la masa inercial y este resultado era interesante porque a través de $\vec{\sigma}$ parecía mostrarse la dependencia explícita del aumento de la masa inercial en el resto de la materia. Con esto en mente pasamos directamente a las observaciones de Brans. Veamos:

Para dejar a un lado el arrastre de los marcos inerciales (que dependen de la aceleración de la materia) y concentrarse en el aumento de la masa inercial, Brans escoge un ejemplo sencillo. Imagina el movimiento de una masa de prueba muy próxima a una pequeña masa $m$ que se encuentra en reposo en el origen. Ambas dentro de un cascarón esférico de masa Ms y radio Rs. Además el cascarón está en reposo. Con lo cual, al aplicar los resultados de Einstein al ejemplo en cuestión, la ecuación de las geodésicas (1) se convierte en:

$$
\frac{d}{d x^{o}}\left[\left(1+\frac{K M_{s}}{8 \pi R_{s}}+\frac{k m}{8 \pi r}\right) v^{i}\right]=\frac{k m}{8 \pi} \frac{\partial}{\partial x^{i}}\left(\frac{1}{r}\right)
$$

Como bien señala Brans el término $k \int \sigma d V / r\left(1+\frac{K M_{s}}{8 \pi R_{s}}+\frac{k m}{8 \pi r}\right)$ es un coeficiente multiplicativo de la aceleración de coordenadas de la partícula de prueba, semejante al coeficiente de resistencia inercial que aparece en la segunda ley de Newton y que por tato Einstein asoció, como acostumbra hacerse, con la masa inercial. Ahora bien, si se recuerda, los cálculos de Einstein son válidos a primer orden en $k \int \sigma d V / r$. Esto significa que la correspondiente ecuación geodésica (2) sólo es válida a primer orden en $K M / R$ y $\mathrm{km} / \mathrm{r}$. Pero, como se ve, ésta está expresada en términos de orden superior y por lo tanto, dentro de los límites de aproximación del método utilizado para calcularlos, los términos que debían representar la influencia machiana sobre la masa inercial no pueden ser retenidos consistentemente. O como dice Brans (Brans,1962, p.390):

In other words, the difference between (2) and

$$
\frac{d}{d x^{\circ}} v^{i}=\frac{k m}{8 \pi} \frac{\partial}{\partial x^{i}}\left(\frac{1}{r}\right)
$$

is to small to be retained in view of the approximation made in deriving (2). 
Con lo cual, dentro de la precisión utilizada, el aumento de la masa inercial estimado por Einstein resultaría irrelevante o despreciable para utilizar la terminología acostumbrada.

En todo caso las objeciones a este resultado pueden impugnarse a las limitaciones del método de aproximación empleado para calcularlo. Así que Brans, para superarlas, decide estudiar las ecuaciones de movimiento llevándolas a orden superior. Antes ha preferido escribir la ecuación de movimiento (2) así:

$$
\frac{d}{d x^{0}} v^{i}=\frac{k m}{8 \pi\left[1+(K / 8 \pi)\left(M_{s} / R_{s}+m / r\right)\right]} \frac{\partial}{\partial x^{i}}\left(\frac{1}{r}\right) .
$$

El resultado encontrado por Brans, al obtener una expresión geodésica análoga, pero llevada a orden superior (reteniendo los términos relevantes) fue:

$$
\frac{d}{d x^{o}} v^{i}=\frac{k m}{8 \pi\left[1+\left(5 K M_{s} / 8 \pi R_{s}\right)\right]} \frac{\partial}{\partial x^{i}}\left(\frac{1}{r}\right)
$$

Sin embargo esta expresión, que incluye supuestos términos machianos, que le diferencian de la expresión newtoniana (3) puede ser objetada, como hizo Brans, atendiendo a razones de honda significación en la interpretación de TGR. Para introducirlas será ilustrativo recordar que sólo hasta 1918- tres años después de cristalizar las ecuaciones de campo gravitatorio de TGR -atendiendo a las incisivas objeciones de Kretschmann- Einstein había reconocido el carácter meramente formal de la covariancia general. Esto, a grandes rasgos, conlleva la nada despreciable consecuencia fundamental de que en la Teoría General de la Relatividad las coordenadas son cantidades auxiliares que permiten asignar valores arbitrarios para cada acontecimiento (evento). Es decir, las coordenadas son estampillas para tabular puntos del espacio-tiempo que no tienen ninguna interpretación física relevante a menos que se especifique cómo está anclado el sistema de coordenadas (los puntos) a eventos en el espacio-tiempo. Einstein sabía esto, pero toda la tradición, que no había puesto sus ecuaciones en lenguaje covariante, y las teorías contemporáneas suyas (como la mecánica cuántica) que tampoco lo hacían, habían trasmitido la costumbre de expresar resultados físicamente substanciales en términos de valores de coordenadas y el hábito, que es una fuerza mayor, también se había asentado en TGR, aunque en esta teoría los valores coordenados no tuvieran, en sí mismos, un significado relevante.

Dicho esto se remarcará que tanto la expresión para las geodésicas calculada por Einstein (1), como la corrección a orden superior (4) efectuada por Brans expresan tanto las distancias como sus derivadas temporales (velocidad y aceleración) en términos de coordenadas. Así que atendiendo a una de las premisas fundamentales en la interpretación habitual para las mediciones de tiempos y distancias en TGR, a saber, la identificación del llamado diferencial de tiempo propio, 


$$
\left.d \tau=\left(-g_{\mu v} d x^{\mu} d x^{v}\right)^{\frac{1}{2}} ; \quad \text { (si } \quad d \tau^{2} \geq 0\right)
$$

con el tiempo medido por un reloj asociado al intervalo de coordenadas $D_{T_{\sigma}} T_{\sigma}=0, d x^{u}$ (como por ejemplo, el tiempo medido por un reloj atómico anclado al origen de las coordenadas $x^{u}$ ), Brans busca las correspondientes expresiones teóricamente substanciales. Por esta vía se obtiene un método para relacionar directamente las mediciones del tiempo propio (que llamaremos $x_{p}^{0}$ en nuestro caso) con el tiempo de coordenadas $\left(x^{0}\right)$. Aún queda por establecer la relación entre las distancias propias $\left(x_{p}^{i}\right)$ y las distancias de coordenadas $\left(x^{i}\right)$. La primera tentación pasa, como es costumbre, por asociar distancias entre partículas (separaciones) a las mediciones hechas mediante barras rígidas. Pero estas resultan correctas tan sólo en el caso en que las marcas de medir coinciden simultáneamente desde el punto de vista exclusivo del sistema en reposo anclado a la barra de medir.

Pero estamos tratando ecuaciones de movimiento así que resulta conveniente reducir todas las mediciones en el espacio-tiempo a mediciones hechas mediante algún tipo de reloj. Y Brans emplea precisamente un método basado en esta consideración. No entro en pormenores técnicos sobre el mismo, pero citaré la sucinta descripción que hace de éste (Brans, 1962, p391):

Defining the velocity of light to be a null geodesic, provides the basis for a method of obtaining a "proper" measurement of a "distance" between particles. Specifically, the proper distance between two time-like paths will be taken as one-half the proper time of flight (measured along one path) of a light ray from one path to the other and back again. This provides a coordinate free, if impractical, method for obtaining a measurable, numerical description of the relative motion of two bodies.

Brans encontró que al aplicar este método a la expresión geodésica (4), que comporta la aplicación de las transformaciones

$$
\begin{gathered}
x_{p}^{0} \cong\left[-g_{00}(0)\right]^{\frac{1}{2}} x^{0}, \\
x_{p}^{i} \cong\left[g_{i i}(0)\right]^{\frac{1}{2}} x^{i},
\end{gathered}
$$

entre tiempos y distancias propias y sus respectivas expresiones en coordenadas, daba como resultado la predicción nula del aumento de la masa inercial de una partícula como consecuencia de la presencia de materia en su vecindad. Concretamente, Brans encontró que en la expresión (4) los términos machianos, que dan cuenta de la influencia de la materia circundante en el aumento de la 
masa inercial, desaparecen. Es decir, que la ecuación de las geodésicas corregida a orden superior se reduce a la expresión

$$
\frac{d^{2}}{d x^{02}} x_{p}^{i}=\frac{k m}{8 \pi} \frac{\partial}{\partial x_{p}^{i}}\left(\frac{1}{r_{p}}\right)
$$

que, como se ve, al ser escrita libre ya de oscuros efectos de coordenadas, no es mas que la aquí llamada expresión newtoniana (3), mostrando así que el aumento de la masa inercial sostenido controversialmente en TGR durante cuatro décadas había sido una quimera.

\section{CONCLUSIÓN}

La evidente lección, lentamente asimilada y olvidada tantas veces por quienes se enfrentan a problemas relacionados con TGR, como el propio Einstein, no era otra que el riesgo y la dificultad que conlleva la costumbre de suscribir significado intrínseco, real, a las cantidades expresadas en coordenadas. Tiempo atrás, en 1917, antes de empezar a ceder a la resignación con respecto al Principio de Mach, Einstein había intentado impugnar el universo de De Sitter argumentando la presencia de una singularidad. Poco mas de un año después Einstein validaba el modelo de De Sitter ante la evidencia de que se trataba simplemente de una singularidad de coordenadas carente de significado intrínseco. En esta ocasión ya no estaba para ser testigo. La moderna formulación intrínseca de TGR previene, en cierta medida, estos riesgos y aunque el comentario pueda colorearse de un matiz anacrónico se dirá que en parte por esto hoy solemos preferir escribir la ecuación de las geodésicas de TGR,

$$
D_{T_{\sigma}} T_{\sigma}=0,
$$

libre de la tara de las coordenadas, pero en algún momento hay que volver a ellas, ya sea para expresar resultados inteligibles o para ajustar modelos a predicciones experimentales. El aumento de la masa inercial comporta un buen ejemplo sobre la frecuente dificultad interpretativa de la covarianza general. Quizá el rasgo más distintivo de TGR.

El aumento de la masa inercial (al igual que alguna variación impugnable en la equivalencia entre masa inercial y masa gravitatoria) nunca había sido detectado. $Y$ el resultado de Brans, tras alguna discusión consecuente, se convirtió en una razón concluyente para dejar de hacerlo. Así que, contrario al optimista parecer de Einstein, el aumento de la masa inercial no resultó ser un efecto real sino, como se ha dicho, un sutil efecto de coordenadas. Empero, a su lado, el arrastre de los marcos inerciales sí es considerado un efecto real y la última tecnología experimental se prepara para detectarlo como una prueba más de la precisión 
predictiva de TGR y de la probable, aunque discutible, cristalización de las ideas de Mach sobre la inercia ${ }^{7}$.

\section{AGRADECIMIENTOS}

Deseo agradecer al doctor Carl Hoefer de la Universidad Autónoma de Barcelona por su atenta lectura comentada de este artículo.

\section{REFERENCIAS}

[1] Barbour, J.(1992) "Einstein and Mach's Principle", En Eisenstadt J. y Kox, A.(eds) Studies in the History of General Relativity, vol 3 de Einstein Studies.pp.125-153. Birkhäuser Boston.

[2] Barbour, J. y Pfister, H. (eds)(1995) Einstein's Studies, vol 6: Mach's Principle: From Newton's Bucket to Quantum Gravity. Birkhäuser. Boston.

[3] Brans, C. (1962) Mach's Principle and the Locally Measured Gravitational Constant in General Relativity, Physical Review, 125,pp 388-396.

[4] Brans, C. y Dicke, R.H. (1962) Mach's Principle and a Relativistic Theory of Gravitation Physical Review, 124,pp 925-930.

[5] Brill, D. R. y Cohen, J. M. (1966) Rotating Masses and their Effect on Inertial Frames,Physical Review, 143,pp 1011-1015.

[6] Cohen, J. M. y Brill, D. R. (1968) Further Examples of Machian Effects of Rotating Bodies in General Relativity,Nuovo Cimento, 56B,pp 209-219.

[7] Einstein, A.(1916) Die Grundlage der allgemeinen Relativit"atstheorie, Annalen der Physik, 49,(translation published in:The Principle of Relativity, collection of papers by A. Einstein et al., New York: Dover[1952],pp 111-164.

[8] Einstein, A. 1917) Cosmological Considerations on The General Theory of Relativity, En: The principle Relativity.pp.177-188.Dover. New York. 1952.

[9] Einstein, A.(1922) The Meaning of Relativity. Methuen. London.

[10] Einstein, A(1949) Autobiographical Notes, En Schilp, P.A.(ed)Albert Einstein: Philosopher-Scientist.pp.1-95.Open Court. La Salle, Illinois.

7 En este sentido véanse en http://einstein.stanford.edu, detalles sobre Gravity Probe-B, un experimento desarrollado por la NASA y la universidad de Stanford destinado a verificar, de acuerdo con TGR, el arrastre inducido por la rotación de la tierra sobre 4 giroscopios puestos a orbitar en un satélite a 400 millas de altura directamente sobre los polos terrestres. 
[11] F"oppl,A.(1904) "Uber absolute und relative Bewegung, Akademie der wissenschaften. Mathematisch-physikalische Klasse. Sitzungsberichtr, 34, pp 5-28.

[12] Friedlander, B. and Friedlander, I. (1896) "Uber absolute und relative Bewegung, Akademie der wissenschaften. Mathematisch-physikalische Klasse. Sitzungsberichtr, 34, pp 5-28.

[14] Hoefer, C.(1994) Einstein's Struggle for a Machian Gravitation Theory, Studies for the History and Philosophy of Science, Vol. 25, 3,pp 287-335.

[15] Mach, E.(1883) The Science of Mechanics. Trad. de T.J Mc Cormack. Open Court. La Salle,Illinois. 1960.

[16] Seeliger,H.(1895)Ueber das Newton'sche Gravitionsgesetz, Astronomische Nachrichten, 137, pp 129-136.

[17] Spezialli, P. (Ed.)(1972)En Albert Einstein, Michel Besso: Correspondance 1903- 1955 Hermann. Paris.

[18] Thirring, H.(1918) "Uber die Wirkung rotierender, ferner Massen in der Einsteinschen Gravitationstheorie,Physikalische Zeitschrift, 19,pp 33-39. 